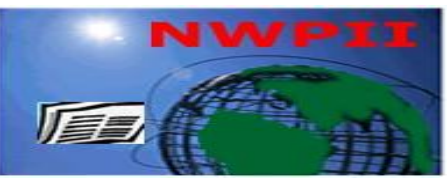

American Journal of Biomedical Sciences

ISSN: 1937-9080

nwpii.com/ajbms

\title{
Approximation of ECG Signals Using Chebyshev Nodes and Lagrange- interpolation
}

\author{
Yadav Om Prakash, Ray Shashwati
}

Department of Electronics and Telecommunication Engineering, Bhilai Institute of Technology, Durg, Chhattisgarh, India

"Corresponding Author

Om Prakash Yadav

Bhilai Institute of Technology Durg

Chhattisgarh

India

Email: omprakashelex@gmail.com

Received:22 November 2018 ; | Revised:30 January 2019 ; | Accepted: 03 June 2019

\section{Abstract}

An ECG (Electrocardiography) is a graphical representation of electrical behavior of heart and is measured by placing electrodes on specific locations of limbs and chest. The characteristics of these signals change during acquisition and transmission due to addition of noises of variable frequency and amplitude. These noises need to be removed for better clinical evaluation. In this paper, ECG signals of MIT-BIH database have been approximated through a series of steps, i.e., denoising using total variation, segmentation using Bottom Up approach and finally utilizing Chebyshev nodes for Lagrange interpolation method. This paper also explores the concept and the benefit of second difference total variation over the first difference. The performance of the method is analyzed in terms of mean absolute deviation, root mean square deviation, percentage root mean square difference error, signal to noise ratio and cross correlation. The results obtained are found to be better than exiting techniques.

Keywords: ECG signal; Total Variation Denoising; Majorization-Minorization optimization; Chebyshev Nodes; Lagrange Interpolation

\section{Introduction}

An ECG is an important tool for cardiac related treatments. The signal is acquired through 16 conventional 12-lead ECG in which ten electrodes are placed on the patient's limbs and chest. The resultant signal ${ }^{[1,2]}$ consists of waves viz., $\mathrm{P}, \mathrm{QRS}, \mathrm{T}$ and $\mathrm{U}$ of different shapes, amplitudes and frequencies which are shown in Figure 1.

The characteristics of these signals are changed due to power line interference (PLI), baseline drift, electrode contact noise, motion artifacts, muscle contraction, instrumentation noise etc ${ }^{[3]}$. Thus, it is necessary to reduce noises from ECG signals up to the extent where ECG retains diagnostic features ${ }^{[4]}$. 


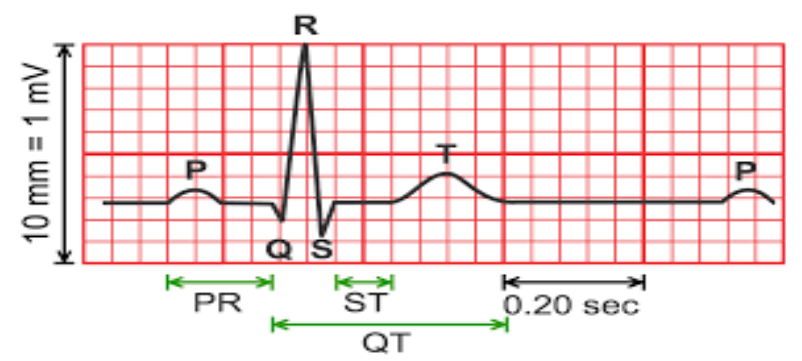

\section{Figure 1: A standard ECG signal with components waves and intervals on ECG grid}

Since, noises introduced in ECG signals are of variable frequency and amplitude, significant noise removal cannot be achieved using a single filter. Traditional analog and digital filters were found to suppress ECG components near to $50 \mathrm{~Hz}$ frequency. Different types of infinite impulse response filters (IIR) and finite impulse response filters (FIR) with unacceptably long transient time were widely used to reduce PLI noises ${ }^{[5,6,7]}$. Also determination of cut-off frequency for these filters was not so easy. Adaptive filters minimize the error between noisy ECG and a reference ECG with high transient time especially on the QRS complex ${ }^{[8]}$. Other filters like least mean square (LMS), normalized least mean square (NLMS), transform domain least mean square (TDLMS) which suffered from numerical instability were also found in literature ${ }^{[9]}$. Baseline wandering is reduced to significant level using linear and polynomial filtering. Low frequency noises were reduced using median filters in ${ }^{[10]}$. Wavelet transform which allows analysis of signal in both time and frequency scales were also find application in ECG denoising. Various ECG filters using wavelet transform can be found in ${ }^{[11,12,13,14]}$. Selection of threshold and decomposition level is still a challenge. Neural networks and genetic algorithms were also applied to reduce noise from ECG signals ${ }^{[15]}$. An effective ECG enhancement technique using total variation was proposed in ${ }^{[16]}$. In this paper, noises present in ECG signals of MIT$\mathrm{BIH}$ are reduced through first difference and second difference total variation. The effect of regularization parameters on these algorithms is also explored. Characterization of these signals through Lagrange-Chebyshev interpolants is also presented and is found to be more useful.

The rest part of the paper is organized as follows: in section 2, we present the total variation denoising technique based on first and second difference. In section 3, we give a brief introduction to characterization using Lagrange-Chebyshev interpolants. In section 4 , we describe the method and discuss the results obtained. In the last section, we give the conclusions regarding the presented approach.

\section{Total variation denoising: First difference- Second difference}

The total variation (TV) of $\mathrm{N}$ point discrete $\operatorname{signalx}(\mathrm{n}), 1 \leq \mathrm{n} \leq \mathrm{N}$ is defined as ${ }^{[17]}$

$\operatorname{TV}(\mathrm{x})=\sum_{\mathrm{n}=1}^{\mathrm{N}}|\mathrm{x}(\mathrm{n})-\mathrm{x}(\mathrm{n}-1)|$

The TV of a signal measures sum of deviations, i.e., difference between consecutive samples of the signal. It is found that signals with high noise have high TV. Therefore, reducing the TV of the signal removes unwanted detail and preserves important diagnostic features ${ }^{[18]}$.

Total variation denoising (TVD) assumes that the noisy data $\mathrm{x}(\mathrm{n})$ is of the form

$$
\mathrm{x}(\mathrm{n})=\mathrm{y}(\mathrm{n})+\mathrm{w}(\mathrm{n}) \quad \mathrm{n}=0, \ldots, \mathrm{N}
$$

where $y(n)$ an approximately piecewise constant signal and $w(n)$ is white Gaussian noise. TVD is defined as an optimization problem which minimizes the cost function (3) for reduction of noises and preservation of sharp edges ${ }^{[19]}$.

$$
\begin{aligned}
& \underbrace{\arg \min }_{\mathrm{x}}=\left\{\mathrm{f}(\mathrm{x})=\frac{1}{2} \sum_{\mathrm{n}=0}^{\mathrm{N}-1}|\mathrm{y}(\mathrm{n})-\mathrm{x}(\mathrm{n})|^{2}+\right. \\
& \lambda \mathrm{n}=1 \mathrm{~N}-1 \mathrm{xn}-\mathrm{x}(\mathrm{n}-1)
\end{aligned}
$$

The first term in (3) represents the mean square error between the observed and the reconstructed signal, and the second term refers to the total variation. The regularization parameter $\lambda$ controls the degree of smoothing with larger value for large noise. Increasing $\lambda$ gives more weight to the TV of the signal ${ }^{[20]}$.

Since ECG signals are not piece-wise constant, TVD using first order differences has the tendency 
to introduce a staircase effect leading to small flat regions in the denoised signal. While conventional TVD may be suitable for filtering piecewise constant signals, it is not usually the best denoising method for more general piecewise-smooth signals ${ }^{[21]}$. For such signals, a higher-order difference can preferably be used instead of the first order differences. So, a variant of TVD is proposed in this paper which would reduces the staircase effect while retaining the quality of the reconstructed signal.

Let the ECG signal $\mathrm{x}$ be represented as $\mathrm{N}$ point vector

$$
x(n)=[x(0), \ldots, x(N-1)]
$$

Expressing the TV of the signal $\mathrm{x}(\mathrm{n})$, in terms of second order differences as

$$
\mathrm{TV}(\mathrm{x})=\sum_{\mathrm{n}=2}^{\mathrm{N}-1} \mid\{\mathrm{x}(\mathrm{n})-\mathrm{x}(\mathrm{n}-1)\}-
$$

$\{\mathrm{xn}-1-\mathrm{x}(\mathrm{n}-2)\}$

Therefore, the optimization problem reduces to

$$
\underbrace{\arg \min }_{\mathrm{x}}=\left\{\mathrm{f}(\mathrm{x})=\frac{1}{2} \sum_{\mathrm{n}=0}^{\mathrm{N}-1}|\mathrm{y}(\mathrm{n})-\mathrm{x}(\mathrm{n})|^{2}+\right.
$$

$\lambda \mathrm{n}=2 \mathrm{~N}-1 \mathrm{xn}-\mathrm{xn}-1-\{\mathrm{xn}-1-\mathrm{x}(\mathrm{n}-2)\}$ (6)

Our aim is to estimate $\mathrm{y}(\mathrm{n})$ from $\mathrm{x}(\mathrm{n})$ by minimizing (6). Since 11 norm is not differentiable, we minimize the objective function using Majorization-Minimization (MM) algorithm.

The MM method solves the optimization problem by replacing a complicated problem by a sequence of simpler problems. Convergence is guaranteed by requiring that the approximating functions majorize the original function at the current solution ${ }^{[22]}$. If $\mathrm{x}, \mathrm{y} \in \mathrm{R}, \mathrm{f}$ and $\mathrm{g}$ be real valued functions on $R^{n}$, then the function $g$ majorizes the function $\mathrm{f}$ at $\mathrm{y}$ if:

(a) $g(x) \geq f(x)$ for all $x$

(b) $\mathrm{g}(\mathrm{y})=\mathrm{f}(\mathrm{y})$

While minimizing the objective function $f$ iteratively, let $\mathrm{x}(\mathrm{k})$ be the current best minimizer at the $\mathrm{k}^{\text {th }}$ iteration. A majorizing function $\mathrm{g}$ is constructed that majorizes $\mathrm{f}$ at $\mathrm{x}(\mathrm{k})$. If $\mathrm{x}(\mathrm{k})$ minimizes $\mathrm{g}$, the procedure is terminated otherwise a new solution $\mathrm{x}(\mathrm{k}+1)$ is found by minimizing $\mathrm{g}$,

$$
\mathrm{f}\left(\mathrm{x}^{\mathrm{k}+1}\right) \leq \mathrm{g}\left(\mathrm{x}^{\mathrm{k}+1}\right) \leq \mathrm{g}\left(\mathrm{x}^{\mathrm{k}}\right)
$$

A new majorizing function is constructed at $\mathrm{x}(\mathrm{k}+1)$, and the steps are repeated to produce a decreasing sequence of function values. In order to construct a majorizer for the objective function given by (6), the property of quadratic majorizers has been exploited. The function $\mathrm{f}(\mathrm{x})=|\mathrm{x}|$ has a quadratic majorizer at each $\mathrm{x}_{\mathrm{k}}$ except at $\mathrm{x}_{\mathrm{k}}=0$. If $\mathrm{x}(\mathrm{k}) \neq 0$ then the majorizer for $\mathrm{f}(\mathrm{x})$ is given by ${ }^{[22]}$

$$
|x|=\frac{1}{2\left|x_{k}\right|} x^{2}+\frac{1}{2}\left|x_{k}\right|
$$

Therefore, instead of minimizing the cost function $f(x)$ directly, the Majorization approach solves a sequence of optimization problems, $\mathrm{G}_{\mathrm{k}}(\mathrm{x})$, $\mathrm{k}=0,1,2, \ldots$, where each function $\mathrm{G}_{\mathrm{k}}(\mathrm{x})$, is a majorizer of $f(x)$. Using (8) we can construct the majorizer for (6) as

$$
\begin{gathered}
\mathrm{G}_{\mathrm{k}}(\mathrm{x})= \\
\frac{1}{2} \sum_{\mathrm{n}}|\mathrm{y}(\mathrm{n})-\mathrm{x}(\mathrm{n})|^{2}+\frac{1}{2} \lambda \sum_{\mathrm{n}}\left[\frac{(\mathrm{x}(\mathrm{n})-\mathrm{x}(\mathrm{n}-1))^{2}}{\left|\mathrm{x}_{\mathrm{k}}\right|}+\left|\mathrm{x}_{\mathrm{k}}\right|\right]
\end{gathered}
$$

Since common ECG contaminants are nonstationary and temporally correlated, time-varying dynamic models are required for the generation of realistic noises.

\section{Characterization using Chebyshev- Lagrange interpolation}

The Chebyshev polynomials of first kind, degree $n$ are defined ${ }^{[23]}$ as:

$$
\mathrm{T}_{\mathrm{n}}(\mathrm{x})=\cos \left(\mathrm{n} \cos ^{-1}(\mathrm{x})\right) \text { for } \mathrm{n} \geq 1
$$

The nth degree Chebyshev polynomial has $\mathrm{n}+1$ zeros (nodes or points) in the interval $[-1,1]$, which can be calculated as:

$$
\mathrm{x}_{\mathrm{j}}=\cos \left(\frac{2 \mathrm{j}+1}{2(\mathrm{n}+1)} \pi\right) \text { for } 0 \leq \mathrm{j} \leq \mathrm{n}
$$

The Chebyshev polynomials are orthogonal in the interval $[-1,1]$ over the weight $\mathrm{w}(\mathrm{x})=$ $\left(1-x^{2}\right)^{-1 / 2}$. Other properties of Chebyshev polynomials can be found in ${ }^{[24]}$. Chebyshev interpolation produces a sequence of polynomials $p(x)$ that converge uniformly to $f(x)$ over $[-1,1]^{[25]}$. If $f(x)$ is a continuous function on $[-1,1]$, the polynomial interpolation of degree $n$ can be obtained by interpolating between the values of $\mathrm{f}(\mathrm{x})$ at $\mathrm{n}+1$ significant points in the interval. Let $\mathrm{f}\left(\mathrm{x}_{\mathrm{j}}\right), \quad 0 \leq \mathrm{j} \leq \mathrm{n} \quad$ be a set of $\mathrm{N}+1$ numbers representing the samples of ECG sequence vector of length $\mathrm{N}$ in $[-1,1]$. Then there exists a unique 
polynomial $\mathrm{p}$ of degree $\mathrm{n} \leq \mathrm{N}$ that interpolates these data, i.e., $p\left(x_{j}\right) \approx f\left(x_{j}\right)$ for each $j$.

If the interpolating polynomial is $c_{n} x^{n}$

$$
\mathrm{p}_{\mathrm{n}}(\mathrm{x})=\mathrm{c}_{0}+\mathrm{c}_{1} \mathrm{x}+\mathrm{c}_{2} \mathrm{x}^{2}+\cdots+\mathrm{c}_{\mathrm{n}-1} \mathrm{x}^{\mathrm{n}-1}+
$$

We require that

$$
c_{0}+c_{1} x_{j}+c_{2} x_{j}^{2}+\cdots+c_{n-1} x_{j}^{n-1}+
$$

$c_{n} x_{j}^{n}=f\left(x_{j}\right)$

In matrix form (15) can be rewritten as

$$
\left[\begin{array}{ccccc}
1 & x_{0} & \Lambda & x_{0}^{n-1} & x_{0}^{n} \\
1 & x_{1} & \Lambda & x_{1}^{n-1} & x_{1}^{n} \\
\mathrm{M} & \mathrm{M} & \Lambda & \mathrm{M} & \mathrm{M} \\
1 & x_{n} & \Lambda & x_{n}^{n-1} & x_{n}^{n}
\end{array}\right]\left[\begin{array}{c}
c_{0} \\
c_{1} \\
\mathrm{M} \\
c_{n}
\end{array}\right]=\left[\begin{array}{c}
f\left(x_{0}\right) \\
f\left(x_{1}\right) \\
\mathrm{M} \\
f\left(x_{n}\right)
\end{array}\right]
$$

In order for the system (14) to have a unique solution, the Vandermonde determinant on the extreme left should be non singular ${ }^{[26]}$. The Vandermonde determinant equals the product of the terms $\left(x_{i}-x_{j}\right)$ for $i>j$, therefore the points $\mathrm{x}_{0}, \ldots \mathrm{x}_{\mathrm{n}}$ should be distinct for the determinant to be non zero. Setting the coefficients as the interpolated values $\mathrm{f}\left(\mathrm{x}_{\mathrm{j}}\right) ; 0 \leq \mathrm{j} \leq \mathrm{n}$, we can write the interpolating polynomial of degree $\mathrm{n}$ using Lagrange's formula[27,28] as

$$
p_{n}(x)=\sum_{j=0}^{n} l_{j}^{n}(x) f\left(x_{i}\right)
$$

where $l_{j}^{n}$ are $(n+1)$ Lagrange polynomials of degree $\leq \mathrm{n}$.

$$
l_{j}^{n}(x)=\prod_{j=0, j \neq i}^{N} \frac{x-x_{j}}{x_{i}-x_{j}}
$$

If $\mathrm{f}(\mathrm{x})$ is $\mathrm{n}+1$ times continuously differentiable in $[-1,1]$, then the interpolation error $\mathrm{E}_{\mathrm{n}}(\mathrm{x})$ is ${ }^{[29]}$

$$
f(x)-p_{n}(x)=\frac{1}{n+1} f^{(n+1)}(\xi) \prod_{j=0}^{n}(x-
$$

$x j, \quad \xi \in[-1,1]$

Therefore,

$\left|E_{n}(x)\right| \leq$

$\frac{1}{(n+1) !}\left|\prod_{j=0}^{n}\left(x-x_{j}\right)\right| \max _{-1 \leq \xi \leq 1}\left|f^{(n+1)}(\xi)\right|$

To minimize the upper bound for $\left|\mathrm{E}_{\mathrm{n}}(\mathrm{x})\right|$, we can minimize the product $\prod_{\mathrm{j}=0}^{\mathrm{n}}\left(\mathrm{x}-\mathrm{x}_{\mathrm{j}}\right)$

If we are free to choose the interpolating points $\mathrm{x}_{0}, \ldots, \mathrm{x}_{\mathrm{n}}$ within this interval, then the product $\prod_{\mathrm{j}=0}^{\mathrm{n}}\left(\mathrm{x}-\mathrm{x}_{\mathrm{j}}\right)$ can be minimized. A better choice of interpolating points $\mathrm{x}_{0}, \ldots, \mathrm{x}_{\mathrm{n}}$ to ensure uniform convergence is the set of zeros of the Chebyshev polynomial $\mathrm{T}_{\mathrm{n}+1}(\mathrm{x})$, instead of equally spaced nodes ${ }^{[27]}$. The following theorem gives an estimate of the error for the above case.

Theorem 1: Assume that $\mathrm{p}_{\mathrm{n}}(\mathrm{x})$ is the Lagrange polynomial that interpolates $\mathrm{f}(\mathrm{x})$ at $\mathrm{x}_{0}, \ldots, \mathrm{x}_{\mathrm{n}}$. Also assume that these $n+1$ interpolation points are the $(\mathrm{n}+1)$ roots of the Chebyshev polynomial $\mathrm{T}_{\mathrm{n}+1}(\mathrm{x})$, given by (11). Then $\forall x \in[-1,1]$

$$
\begin{aligned}
& \left|f(x)-p_{n}(x)\right| \leq \\
& \frac{1}{2^{n}(n+1) !} \max _{-1 \leq \xi \leq 1}\left|f^{(n+1)}(\xi)\right|
\end{aligned}
$$

If the interpolation interval for the function $\mathrm{f}(\mathrm{x})$ is $x \in[a, b]$, we transform the interval $y \in[-1,1]$ using

$$
\mathrm{x}=\frac{(\mathrm{b}-\mathrm{a}) \mathrm{y}+(\mathrm{a}+\mathrm{b})}{2}
$$

This converts the interpolation problem for $f(x)$ on $[a, b]$ into interpolation problem for $\mathrm{f}(\mathrm{x})=$ $\mathrm{g}(\mathrm{x}(\mathrm{y}))$ in $\mathrm{y} \in[-1,1]$. The Chebyshev points in the interval $y \in[-1,1]$ are the roots of the Chebyshev polynomial $\mathrm{T}_{\mathrm{n}}(\mathrm{y})$, i.e,

$$
y_{j}=\cos \left(\frac{2 j+1}{2(n+1)} \pi\right) \quad 0 \leq j \leq n
$$

The corresponding $\mathrm{n}+1$ interpolation points in the interval $[a, b]$ using (21) are now

$$
\mathrm{x}_{\mathrm{j}}=\frac{(\mathrm{b}-\mathrm{a}) \mathrm{y}+(\mathrm{a}+\mathrm{b})}{2} 0 \leq \mathrm{j} \leq \mathrm{n}
$$

The interpolation error now is given by

$\left|f(x)-p_{n}(x)\right|=$

$\frac{1}{2^{\mathrm{n}}(\mathrm{n}+1) !}\left|\frac{\mathrm{b}-\mathrm{a}}{2}\right|^{\mathrm{n}+1} \max _{-1 \leq \xi \leq 1}\left|\mathrm{f}^{(\mathrm{n}+1)}(\xi)\right|$

where $p_{n}(x)$ is the Lagrange interpolating polynomial based on Chebyshev nodes. Delving deeper into the advantages of using Chebyshev interpolating nodes, we observe that Runge phenomenon does not occur with the effect that the error tends to decrease with the increasing degree of the Lagrange Chebyshev interpolating polynomial, whereas the same may not be true for equally spaced nodes. Alternatively, we can express the nth degree interpolating polynomial $\mathrm{p}_{\mathrm{n}}(\mathrm{x})$ as a sum of Chebyshev polynomials $\mathrm{T}_{\mathrm{k}}\left(\mathrm{x}_{\mathrm{j}}\right)^{[30]}$.

$$
\mathrm{p}_{\mathrm{n}}(\mathrm{x})=\sum_{\mathrm{k}=0}^{\mathrm{n}} \mathrm{c}_{\mathrm{k}} \mathrm{T}_{\mathrm{k}}(\mathrm{x}), \quad \mathrm{x} \in[-1,1]
$$

where the coefficients ck are defined as

$$
c_{k}=\frac{2}{n+1} \sum_{j=0}^{n} f\left(x_{j}\right) T_{k}\left(x_{j}\right), \quad k=0, \ldots, n
$$

where

$$
x_{j}=\cos \left(\frac{(2 j+1) \pi}{2(n+1)}\right) j=0, \ldots, n
$$


Let

$$
x=\theta, \theta \in[-\pi, \pi]
$$

then,

$c_{k}=\frac{2}{n+1} \sum_{j=0}^{n} g\left(\theta_{j}\right) \cos \left(k \theta_{j}\right) \quad k=1, \ldots, n$

with

$$
\theta_{\mathrm{j}}=\frac{(2 \mathrm{j}+1) \pi}{2(\mathrm{n}+1)}
$$

Replacing $f(\cos \theta)$ by a periodic

function $g(\theta)$,

$$
c_{k}=\frac{2}{n+1} \sum_{j=0}^{n} g\left(\theta_{j}\right) \cos \left(k \theta_{j}\right), k=0, \ldots, n
$$

Thus $c_{k}$ is discrete approximation to the Fourier series coefficients

$$
c_{\mathrm{i}}^{\mathrm{s}} \approx \frac{1}{\pi} \int_{-\pi}^{\pi} \mathrm{g}(\theta) \cos (\mathrm{k} \theta) \mathrm{d} \theta
$$

Applying the trapezoidal rule approximation

$$
\mathrm{c}_{\mathrm{i}}^{\mathrm{S}}=\frac{1}{\pi} \frac{\pi}{(\mathrm{n})} \sum_{\mathrm{k}=0}^{\mathrm{n}+1} \mathrm{~g}\left(\frac{(2 \mathrm{j}+1) \pi}{2(\mathrm{n}+1)}\right) \cos \left(\frac{(2 \mathrm{j}+1) \pi}{2(\mathrm{n}+1)}\right)
$$

which is same as (30) for $\mathrm{c}_{\mathrm{k}}$.

Thus (32) is equivalent to the discrete Fourier transform of the transformed function $\mathrm{g}(\theta)=$ $\mathrm{f}(\cos \theta)$. Chebyshev interpolation can effectively be considered as the partial sum of the approximate Chebyshev series expansion obtained by replacing the Fourier transform $\mathrm{c}_{\mathrm{k}}^{\mathrm{s}}$ by discrete Fourier transform $c_{k}$.

\section{Methods and Results}

Presence of noises degrade the signal quality and changes the morphology of the ECG signal, thus establishing noise removal is an essential step in ECG preprocessing for better performance, analysis and diagnosis. In this paper, first difference, second difference and LagrangeChebyshev interpolants are used for noise removal from ECG signals. To test and to compare the performance of the algorithms, we perform all the algorithms in Matlab environment. The signals used for analysis are taken from MIT-BIH [31] arrhythmia database. Each record consists of two channels of signals, which are of 10 seconds duration with 3600 samples, sampled at a rate of $360 \mathrm{~Hz}$ with 11 bits per sample of resolution.

\subsection{Computational Performance}

Let $x(n)$ be the original signal and $y(n)$ be reconstructed ECG signal of length $\mathrm{N}$. The error between the signals is evaluated as:

$$
e(n)=x(n)-y(n)
$$

The performance of algorithms with respect to the signals is evaluated in terms of following parameters:

\subsubsection{Mean Absolute Deviation}

Mean absolute deviation (MAD) provides average of absolute deviation of reconstructed signal from the original signal.

$$
\operatorname{MAD}=\frac{1}{\mathrm{~N}} \sum_{\mathrm{i}=1}^{\mathrm{N}}|\mathrm{e}(\mathrm{n})|
$$

\subsubsection{Root Mean Square Difference}

The root-mean-square difference (RMSD) measures mean of the differences between reconstructed and the original signal ${ }^{[32]}$. The RMSD is more useful when large errors are particularly undesirable

$$
\operatorname{MSD}=\sqrt{\frac{\left.\sum_{\mathrm{i}=1}^{\mathrm{N}} \mathrm{e}(\mathrm{n})\right|^{2}}{\mathrm{~N}}}
$$

\subsubsection{Percentage Root-Mean-Square Difference}

The percentage root mean square difference ${ }^{[32]}$ (PRD) is calculated by:

$$
\mathrm{PRD}=\sqrt{\frac{\sum_{\mathrm{i}=1}^{\mathrm{N}}|\mathrm{e}(\mathrm{n})|^{2}}{\sum_{\mathrm{i}=1}^{\mathrm{N}}|\mathrm{x}(\mathrm{n})-\overline{\mathrm{x}}|^{2}}}
$$

where $\overline{\mathrm{x}}$ is the mean of the original signal. The PRD is chosen to remove the baseline or to eliminate the dc level which is added to ECG signals for storage purpose.

\subsubsection{Signal to Noise Ratio}

Signal-to-noise ratio (SNR) is defined as the ratio of signal power to the noise power corrupting the signal ${ }^{[32]}$. A ratio higher than 1:1 indicates more signal than noise. 


$$
\operatorname{SNR}(d B)=20 \log _{10} \frac{\sum_{\mathrm{i}=1}^{\mathrm{N}}|\mathrm{x}(\mathrm{n})-\overline{\mathrm{x}}|^{2}}{\sum_{\mathrm{i}=1}^{\mathrm{N}}|\mathrm{e}(\mathrm{n})|^{2}}
$$

\subsubsection{Cross Correlation Coefficients}

A measure that determines the degree to which two variable's signals is associated. Value near to 1 indicates close resemblance between original and reconstructed signal.

$$
\mathrm{CC}=\frac{\mathrm{N}\left(\sum \mathrm{xy}\right)-\left(\sum \mathrm{x}\right)\left(\sum \mathrm{y}\right)}{\sqrt{\left[\mathrm{N} \sum \mathrm{x}^{2}-\left(\sum \mathrm{x}\right)^{2}\right]\left[\mathrm{N} \sum \mathrm{y}^{2}-\left(\sum \mathrm{y}\right)^{2}\right]}}
$$

The higher SNR, CC value and lower MAD, RMSD and PRD values means better signal restoration performance.

\subsection{TVD 1D and TVD 2D ECG signal characterization}

The MM approach to minimize the function $\mathrm{f}(\mathrm{x})$ can be summarized as ${ }^{[18]}$ :
1. Store the noisy ECG signal as data and set the number of data points.

2. Set regularization parameter $\lambda$.

3. Set number of iterations as 30 .

4. Set $\mathrm{k}=0$. Initialize $\mathrm{x}(0)$ as the original signal.

5. Choose $G_{k}(x)$ using (9) such that

(a) $\mathrm{G}_{\mathrm{k}}(\mathrm{x})>f(\mathrm{x})$ for all $\mathrm{x}$

(b) $G_{k}(x)=f\left(x_{k}\right)$

6. Set $x(k+1)$ as the minimizer of $G_{k}(x)$.

Initially we started by taking $\lambda=0.001$.

Results were numerically good but visually poor. So, the value of $\lambda$ is increased slowly and performance parameters were noted. The Table 1 shows performance matrices for different values of $\lambda$ wherein bold, italic and red parameters represent result of second order TVD. From the Table 1 , it is observed that at lower value of $\lambda$, performance TVD 1D is better than TVD 2D whereas at higher values, TVD 2D overtakes TVD $1 \mathrm{D}$.

Table 1: Relation between first order and second order total variation denoising with respect to $\lambda$

\begin{tabular}{|c|c|c|c|c|c|c|c|c|c|c|}
\hline $\begin{array}{c}\lambda \\
\times 10^{(-3)}\end{array}$ & $\begin{array}{c}\text { MAD } \\
\mathrm{x} 10^{(-3)}\end{array}$ & $\begin{array}{c}\text { MAD } \\
\mathrm{x} 10^{(-3)}\end{array}$ & $\begin{array}{c}\text { RMSD } \\
\mathrm{x} 10^{(-2)}\end{array}$ & $\begin{array}{c}\text { RMSD } \\
\mathrm{x} 10^{(-2)}\end{array}$ & PRD & PRD & SNR & SNR & CC & CC \\
\hline 1 & 2 & 4 & 12 & 19 & 0.67 & 1.14 & 50.21 & 45.60 & 1.000 & 0.9999 \\
\hline 5 & 10 & 14 & 42 & 51 & 2.59 & 3.13 & 38.47 & 36.82 & 0.9997 & 0.9995 \\
\hline 10 & 20 & 22 & 66 & 74 & 4.04 & 4.52 & 34.61 & 33.64 & 0.9992 & 0.9990 \\
\hline 50 & 65 & 54 & 22 & 133 & 7.53 & 8.17 & 29.20 & 28.49 & 0.9972 & 0.9967 \\
\hline 100 & 82 & 22 & 147 & 74 & 9.07 & 4.52 & 27.59 & 33.64 & 0.9962 & 0.9990 \\
\hline
\end{tabular}

So, it can be concluded that better results can be achieved for TVD 2D at higher value of $\lambda$.
Results of both TVD 1D and TVD 2D at $\lambda=0.01$ are indicated in Table 2 and 3 respectively.

Table 2: Quality assessment matrix of TVD 1D at $\lambda=0.01$

\begin{tabular}{|c|c|c|c|c|c|}
\hline Signal & MAD & RMSD & PRD & SNR & CC \\
\hline 100 & 0.0200 & 0.6145 & 3.6100 & 35.4131 & 0.9994 \\
\hline 104 & 0.0200 & 0.5294 & 1.9154 & 36.7611 & 0.9998 \\
\hline 108 & 0.0200 & 0.6568 & 4.0377 & 34.6139 & 0.9992 \\
\hline 112 & 0.0200 & 0.4667 & 2.2984 & 45.8739 & 0.9997 \\
\hline 115 & 0.0200 & 0.5075 & 1.7069 & 40.9574 & 0.9999 \\
\hline 117 & 0.0200 & 0.5170 & 2.2271 & 44.5854 & 0.9998 \\
\hline 122 & 0.0200 & 0.6214 & 1.7791 & 43.1285 & 0.9998 \\
\hline 201 & 0.0197 & 0.5116 & 2.5891 & 34.4319 & 0.9997 \\
\hline 205 & 0.0200 & 0.4808 & 2.6060 & 38.6971 & 0.9997 \\
\hline 207 & 0.0200 & 0.6380 & 2.1051 & 35.2116 & 0.9998 \\
\hline 214 & 0.0200 & 0.6309 & 1.3193 & 37.7987 & 0.9999 \\
\hline 220 & 0.0200 & 0.4540 & 1.4573 & 43.3975 & 0.9999 \\
\hline
\end{tabular}


Table 3: Quality assessment matrix of TVD 2D at $\lambda=0.01$

\begin{tabular}{|c|c|c|c|c|c|}
\hline Signal & MAD & RMSD & PRD & SNR & CC \\
\hline 100 & 0.0207 & 0,6755 & 3.9683 & 34.5911 & 0.9992 \\
\hline 104 & 0.0211 & 0.6985 & 2.5274 & 34.3528 & 0.9997 \\
\hline 108 & 0.0222 & 0.7352 & 4.5193 & 33.6351 & 0.9990 \\
\hline 112 & 0.0171 & 0.4616 & 2.2735 & 45.9685 & 0.9997 \\
\hline 115 & 0.0167 & 0.4879 & 1.6410 & 41.2991 & 0.9999 \\
\hline 117 & 0.0202 & 0.5522 & 2.3786 & 44.0140 & 0.9997 \\
\hline 122 & 0.0248 & 0.6920 & 1.9812 & 42.1940 & 0.9998 \\
\hline 201 & 0.0175 & 0.6027 & 3.0501 & 33.0086 & 0.9995 \\
\hline 205 & 0.0181 & 0.4245 & 2.3008 & 39.7792 & 0.9997 \\
\hline 207 & 0,0233 & 0.7586 & 2.5033 & 33.7068 & 0.9997 \\
\hline 214 & 0.0212 & 0.7645 & 1.5988 & 36.1302 & 0.9999 \\
\hline 220 & 0.0198 & 0.4778 & 1.5334 & 42.9549 & 0.9999 \\
\hline
\end{tabular}

More or less the performance parameters of TVD 1D and TVD 2D are same. However, second difference is more suitable as staircase effect is
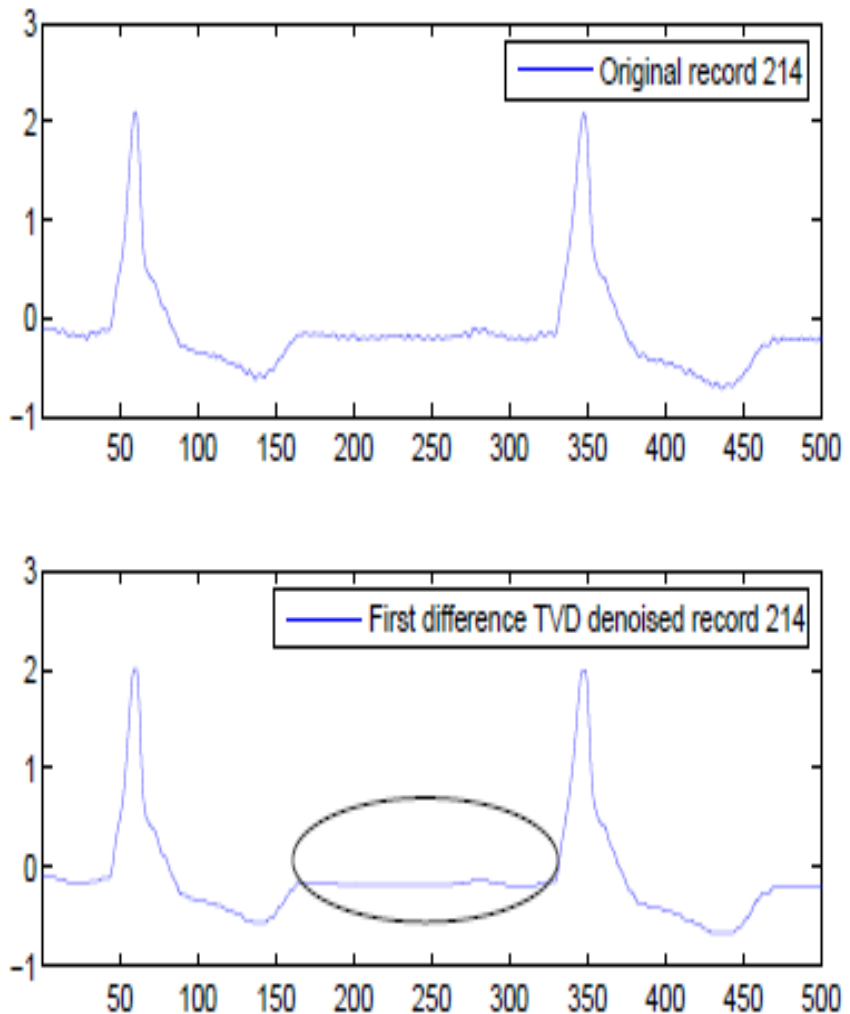

Figure 2: Original and TVD 1D reconstructed record number 214 significantly reduced which can be seen in the Figures 2-3.
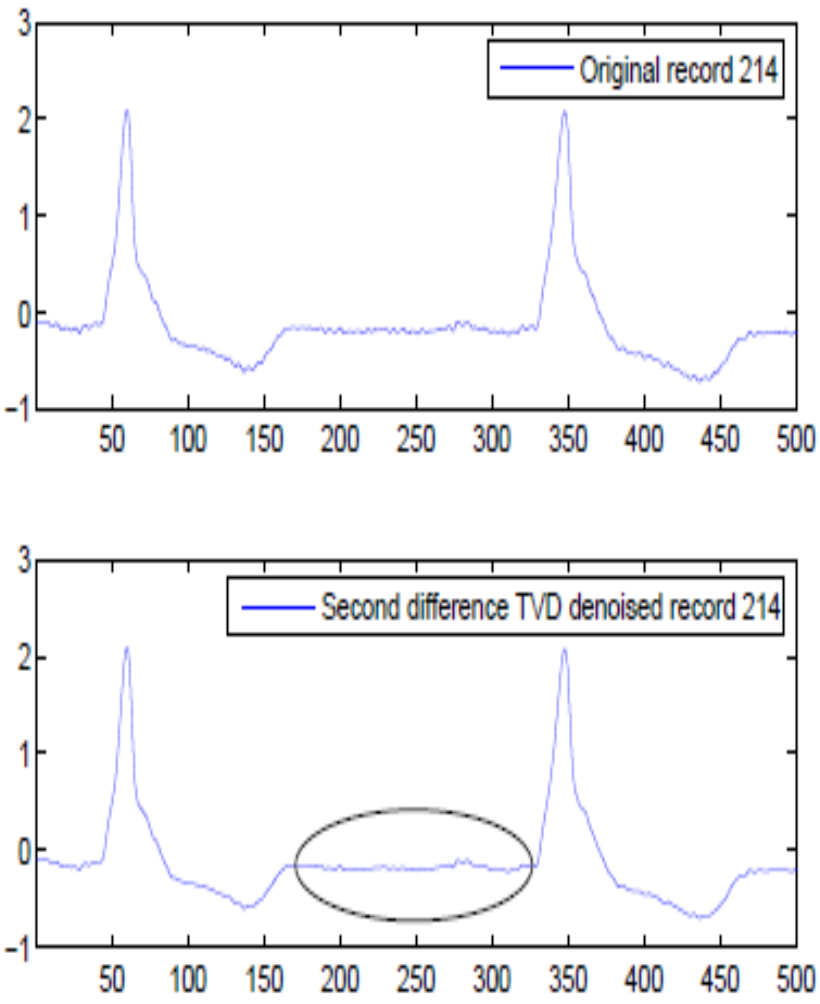

Figure 3: Original and TVD 2D reconstructed record number 214 


\subsection{ECG characterization through Lagrange- Chebyshev interpolation}

Here we need to construct an interpolating polynomial $\mathrm{p}_{\mathrm{n}}(\mathrm{x})$ using (24) with the $\mathrm{N}$ ECG samples using the Chebyshev nodes. Since an ECG signal sampled value may not be available at all the Chebyshev nodes, we derive these values by linear interpolation using adjacent ECG sampled values. We continue increasing the order till our error criterion is met. This approximation technique can be summarized as:

1. Fix the order $n$ and the tolerance $\xi=10^{-4}$ for the Lagrange-Chebyshev polynomial approximation.

2. Transform the Chebyshev nodes on the domain $[a, b]$ and calculate the zeros i.e., the Chebyshev nodes $\mathrm{x}_{\mathrm{j}}$ using (22).

3. Find the function value $f\left(x_{j}\right)$ by linear interpolation using the two adjacent samples around $x_{j}$.

4. Construct interpolating polynomial $\mathrm{p}_{\mathrm{n}}(\mathrm{x})$ using (24).

5. Calculate error $E_{n}(x)=\max \left|f(x)-p_{n}(x)\right|$.
6. If $E_{n}(x)>\xi$ then $n=n+1$ and go to step 2 .

In order to denoise the whole signals, high order polynomials were required which increases computation time. Also errors for this approach were very high. So, to reduce the computation time and error, we segmented the whole signal into suitable (till optimum results are achieved) number of segments. This is done by implementing Bottom up approach. The Bottom up algorithm, also called as iterative merge which begins by dividing the original time series data of length $\mathrm{n}$, into a large number of segments and is consequently merged into bigger segments until stopping criteria is met ${ }^{[33]}$. So, segmentation is done before interpolation. The signal reconstruction stage consists of sequentially appending the segments to obtain the complete reconstructed signal which does not require any selection of significant coefficients. Here the 10 seconds (3600 samples) of original signal from MIT-BIH database is divided into 100 segments and then the individual segments are interpolated by 50th order Lagrange-Chebyshev interpolants. Table 4 shows the results of LagrangeChebyshev interpolation for same set of signals.

Table 4: Quality assessment matrics for Lagrange-Chebyshev interpolation

\begin{tabular}{|c|c|c|c|c|c|}
\hline Signal & $\begin{array}{c}\text { MAD } \\
\times 10^{-6}\end{array}$ & $\begin{array}{c}\text { RMSD } \\
\times 10^{-5}\end{array}$ & PRD & CC \\
\hline 100 & 1.83 & 6.16 & 0.0019 & 44.83 & 0.9977 \\
\hline 104 & 2.89 & 9.55 & 0.0017 & 43.06 & 0.9982 \\
\hline 108 & 0.15 & 1.12 & $1.7153 \times 10^{-4}$ & 66.91 & 1.0000 \\
\hline 112 & 0.80 & 2.87 & $4.6281 \times 10^{-4}$ & 55.97 & 0.9999 \\
\hline 115 & 1.81 & 5.25 & 0.0030 & 50.34 & 0.9945 \\
\hline 117 & 2.59 & 13.00 & 0.0014 & 43.65 & 0.9987 \\
\hline 122 & 0.39 & 11.32 & 0.0023 & 47.61 & 0.9968 \\
\hline 201 & 0.84 & 3.39 & $9.7624 \times 10^{-4}$ & 44.81 & 0.9994 \\
\hline 205 & 0.70 & 3.83 & $1.1696 \times 10^{-4}$ & 53.05 & 1.0000 \\
\hline 207 & 12.01 & 24.68 & 0.0139 & 23.17 & 0.8837 \\
\hline 214 & 5.70 & 16.91 & 0.0090 & 32.29 & 0.9535 \\
\hline 220 & 3.93 & 14.48 & 0.0031 & 41.46 & 0.9941 \\
\hline
\end{tabular}

Although the number of segments has been kept same for all the signals, all the segments cannot be approximated by a single polynomial because they are of unequal sample lengths due to the variable shape of ECG within and across patients. Here our interest is to denoise the signal while retaining its characteristics. So, number of segments is not so important.

The performance matrices for the total variation are more or less similar. RMSD value is of order $10^{-1}$ whereas MAD values are upto $10^{-2}$. Maximum value of PRD is 4 which can be 
considered to be within diagnostic limits. SNR and $\mathrm{CC}$ values are also high indicating high level of noise suppression and high resemblance of timing pattern of reconstructed signal with the original signal. So, both the TVD 1D and TVD 2D methods are suitable for noise suppression of ECG signals. However, visually better results are obtained for TVD 2D. As compared to these techniques, results of Lagrange- Chebyshev are much better. Errors are significantly reduced up to $10^{-4}$. Signal contents
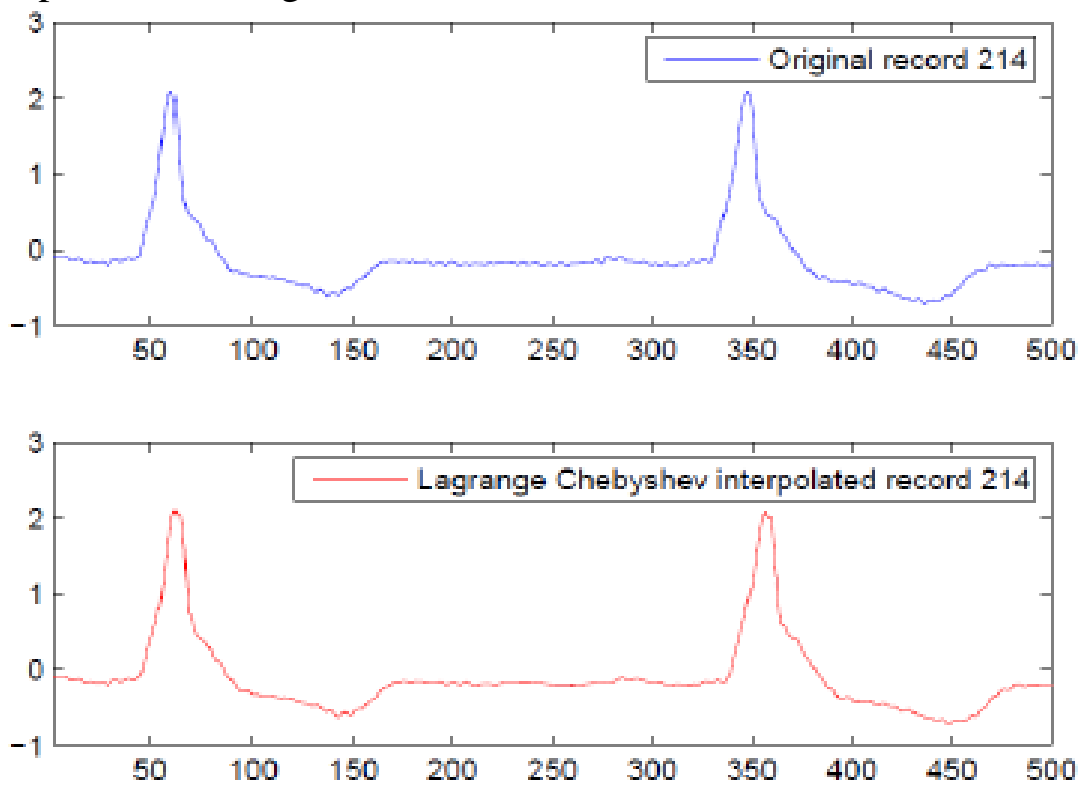

Figure 5: Original and Lagrange -Chebyshev reconstructed record number 214

\section{Conclusion}

ECG signal records voltage potentials of cardiac activity. These signals are often contaminated by various types of noises. In this paper, ECG signals from MIT-BIH database were denoised using TVD 1D, 2D and LagrangeChebyshev interpolation. It is concluded that both methods of TVD are suitable for ECG denoising but TVD 2D results are visually better. Also regularization parameter which controls degree of denoising plays an important role. Same set of signals are denoised using Lagrange- Chebyshev interpolation method of high order and results obtained are comparable. Results are further improved by segmentating ECG signals using Bottom up approach. The results obtained are diagnostically acceptable and are found to be superior to those reported in the existing literature. are also enhanced to high level as compared to noise level. $\mathrm{CC}$ values are found to be less than the methods which can be improved either by increasing number of segments or by increasing order of the polynomial. Original and LagrangeChebyshev approximated record 214 is presented in Figure 4. Thus the proposed method will be more suitable for ECG denoising. 
algorithms. IEEE Trans Biomed Eng 1990; 37(1): 85-98 DOI: $\underline{10.1109 / 10.43620}$

4 A.Bharadwaj and U. Kamath.Techniques for accurate ECG signal processin. EE Times, 2011 Vol. 14, pp. 1-7.

5 Ma WK, Zhang YT, Yang FS. A fast recursive-least-squares adaptive notch filter and its applications to biomedical signals. Med Biol Eng Comput 1999; 37(1): 99-103 DOI:10.1007/BF02513273

6 S. Rani, A. Kaur, J. Ubhi.Comparative study of FIR and IIR filters for the removal of Baseline noises from ECG signal,International Journal of Computer Science and Information Technologies 2011, Vol. 2, No. 3,pp. 1105-1108.

7 Poungponsri, S., X. H. Yu .Electrocardiogram (ECG) signal modeling and noise reduction using wavelet neural networks. IEEE International Conference on Automation \& Logistics IEEE, 2009. pp. 394-398 DOI:10.1109/ICAL.2009.5262892

8 Thakor NV, Zhu YS. Applications of adaptive filtering to ECG analysis: noise cancellation and arrhythmia detection. IEEE Trans Biomed Eng 1991; 38(8): 785-794

DOI: $\underline{10.1109 / 10.83591}$

9 Javed, Shazia , N. A. Ahmad . An adaptive noise cancelation model for removal of noise from modeled ECG signals.IEEE Region 10 Symposium IEEE, 2014, pp. 471-475 DOI: 10.1109/TENCONSpring.2014.6863079

10 J.S. Wang, Y. Zhang, P. Zhang, S.F, Sun.Research on denoising algorithm for ECG signals, IEEE 29th Chinese Control Conference (CCC) 2010, pp. 2936-2940.

11 P.B. Patil and M.S. Chavan.A wavelet basedmethod for denoising of biomedical signal, International Conference on Pattern Recognition, Informatics and Medical Engineering (PRIME), IEEE 2012, pp. 278283 DOI:10.1109/ICPRIME.2012.6208358

12 P. Karthikeyan, M. Murugappan, S. Yaacob. ECG signal denoising using wavelet thresholding techniques in human stress assessment.International Journal on Electrical Engineering and Informatics 2012, Vol.4,No.2, pp. 306-319
13 M. Alfaouri and K. Daqrouq.ECG denoising by sparse wavelet shrinkage. American Journal of Applied Sciences 2008, Vol.5,pp. 276-281.DOI:10.3844/ajassp.2008.276.281

14 B.N. Singh and A.K. Tiwari.Optimal selection of wavelet basis function applied to ECG signal denoising.Digital signal processing 2006 , Vol. 16,pp. 275-287 DOI:10.1016/j.dsp.2005.12.003

15 H.D. Kim, C.H. Min, T.S. Kim.Adaptable noise reduction of ECG signals for feature extraction, International Symposium on Neural Networks. Springer 2006, pp. 58659.DOI:10.1007/11760191_86

16 X. Ning and I.W. Selesnick.ECG enhancement and QRS detection based on sparse derivative, Biomedical Signal Processing and Control 2013, Vol. 8,pp. 713-723. DOI:10.1016/j.bspc.2013.06.005

17 I. Selesnick, Total variation denoising (an MM algorithm). NYU Polytechnic School of Engineering Lecture Notes 2012.

18 O.P. Yadav and S. Ray.Total Variational Denoising of ECG Signals using MajorizationMinorization Technique, Indian Control Conference. IIT Madras, Chennai 2015, pp. 165-169

19 L.I. Rudin, S. Osher, E. Fatemi.Nonlinear total variation based noise removal algorithms. Physica D: Nonlinear Phenomena 1992 Vol. 60,pp. 259-268. DOI:10.1016/01672789(92)90242-F

20 V. Solo.Selection of regularisation parameters for total variation denoising, Proceedings of IEEE International Conference on Acoustics, Speech, and Signal Processing 1999,Vol. 3, pp. 1653-1655.

21 I.W. Selesnick, I. and Bayram, I.Total variation filtering. http://eeweb.poly.edu/iselesni/lecture notes/ TV filtering.pdf 2010 .

22 de Leeuw J, Lange K. Sharp Quadratic Majorization in One Dimension. Comput Stat Data Anal 2009; 53(7): 2471-2484 DOI: 10.1016/j.csda.2009.01.002

23 A. Gil,J. Segura, N. Temme,Numerical Methods for Special Functions, Society for Industrial and Applied Mathematics 2007.DOI: $10.1137 / 1.9780898717822$ 
G. Szego .Orthogonal Polynomials,American Mathematical Society Colloquium Publications: American Mathematical Society Providence, Rhode Island 1975, Vol. 23.

25 J.H. Mathews and K.D. Fink,Numerical methods using MATLAB,Upper Saddle River, NJ 2004, Vol. 4.

26 K. Bjorck, and V. Pereyra,Solution of Vandermonde systems of equations, Mathematics of Computation 1970, Vol. 24,pp. 893-903..DOI:10.2307/2004623

27 W.Y. Yang, W. Cao, T.S. Chung, J Morris, Applied numerical methods using MATLAB,John Wiley \& Sons 2005.DOI: $10.1002 / 0471705195$

28 W.C.C. Chan, C.J. Chyan, H. Srivastava. The Lagrange polynomials in several variables. Integral Transforms and Special Functions 2001, Vol. 12,pp. 139-148. DOI:10.1080/10652460108819340

29 G.M. Caporale and M. Cerrato.Chebyshev polynomial approximation to approximate partial differential equations, Computation Economics 2010, Vol. 35 DOI: $\underline{10.1007 / \mathrm{s} 10614-009-9172-8}$
30 J.C. Mason and D.C. Handscomb.Chebyshev polynomials; CRC Press Company Boca Raton London New York Washington, D.C 2002.DOI:10.1201/9781420036114

31 Goldberger AL, Amaral LA, Glass L, Hausdorff JM, Ivanov PC, Mark RG, Mietus JE, Moody GB, Peng CK, Stanley HE. PhysioBank, PhysioToolkit, and PhysioNet: components of a new research resource for complex physiologic signals. Circulation 2000; 101(23):

E215-220

DOI: $\underline{10.1161 / 01 . C I R .101 .23 . e 215 ~}$

32 Luong, Duong Trong, Nguyen Minh Duc, Nguyen Tuan Linh, Nguyen Thai $\mathrm{Ha}$, and Nguyen Duc Thuan.Advanced Two-State Compressing Algorithm: A Versatile, Reliable and Low-Cost Computational Method for ECG Wireless Applications.American Journal of Biomedical Sciences 2016, Vol. 8(1), pp. 1-23. DOI:10.5099/aj160100001

33 O.P. Yadav and S. Ray. Smoothening and Segmentation of ECG Signals Using Total Variation Denoising - MinimizationMajorization and Bottom-Up Approach. Procedia Computer Science 2015,Vol. 85,pp. 483-489.DOI:10.1016/j.procs.2016.05.195 